\title{
BMC Anesthesiology reviewer acknowledgement, 2014
}

Thomas A Rowles

\section{Contributing reviewers}

The editors of BMC Anesthesiology would like to thank all our reviewers who have contributed their time to the journal in Volume 14 (2014).

Mohamed Abdulatif

Egypt

Serefden Acikgoz

Turkey

Yushi Adachi

Japan

M. Christopher Adams

USA

Massimo Agostoni

Italy

Vassilis Aidinis

Greece

Ibrahim Ozkan Akinci

Turkey

Daniela Alampi

Italy

Giancarlo Albo

Italy

Charles Algert

Australia

Nicole Almenrader

Italy

Michael Andrawes

USA

Christoph Ansorge

Sweden
Richard Applegate

Karim Asehnoune

France

Eftihia Asprodini

Greece

Aimee Aysenne

USA

Aranya Bagchi

USA

Michael Barrington

Australia

Marc Beaussier

France

Helene Beloeil

France

Jan Belohlavek

Czech Republic

Dan Benhamou

France

Monika Berns

Germany

Andrew Bersten

Australia

Azize Bestas

Turkey
Indiradevi Bhagavatula

India

Gianni Biancofiore

Italy

Daniele Biasucci

Italy

Elena Bignami

Italy

Jan Blaha

Czech Republic

Kevin Blaine

USA

Maria Boddi

Italy

Manuela Bonizzoli

Italy

Giovanni Borghi

Italy

Marie Bosman

United Kingdom

Alex Bottle

United Kingdom

Pervin Bozkurt Sutas

Turkey

Barbara Brandom

USA

Correspondence: tom.rowles@biomedcentral.com

BioMed Central, Floor 6, 236 Gray's Inn Road, London WC1X 8HB, UK 


\begin{tabular}{|c|c|c|}
\hline Nicola Brienza & Daniel Chipman & Franklin Dexter \\
\hline Italy & USA & USA \\
\hline Yuriy Bronshteyn & Stephen Choi & Matteo Di Nardo \\
\hline USA & Canada & Italy \\
\hline Bruno Buchholz & Christopher Choukalas & Pierre Diemunsch \\
\hline Argentina & USA & France \\
\hline Eric Busch & Gilda Cinnella & Yalim Dikmen \\
\hline USA & Italy & Turkey \\
\hline John Butterworth & Yann-Erick Claessens & Dawn Dillman \\
\hline USA & Monaco & USA \\
\hline Elif Cadirci & Nathalie Clavier & Ning Ding \\
\hline Turkey & France & China \\
\hline Brian Cairns & Richard Cooper & Sang-Hwan Do \\
\hline Canada & Canada & South Korea \\
\hline Guy Cammu & Menino Osbert Cotta & Marie-Agnès Docquier \\
\hline Belgium & Australia & Belgium \\
\hline Anna Camporesi & Gregory Crosby & William Donaldson \\
\hline Italy & USA & United Kingdom \\
\hline Gianluca Cappelleri & Giovanni Cucchiaro & Anne Donovan \\
\hline Italy & USA & USA \\
\hline Cesar Carcamo & Douglas Curran-Everett & Roberto Dossi \\
\hline Chile & USA & Italy \\
\hline Maria Carmona & J. Randall Curtis & Michael Duggan \\
\hline Brazil & USA & USA \\
\hline Daniel Carr & Wojciech Dabrowski & Roderic Eckenhoff \\
\hline USA & Poland & USA \\
\hline Michele Carron & Aniello De Nicola & Richard Egan \\
\hline Italy & Italy & United Kingdom \\
\hline Davide Cattano & Gennaro De Pascale & Matthias Eikermann \\
\hline USA & Italy & USA \\
\hline Erol Cavus & Nicolas De Prost & Douglas Eleveld \\
\hline Germany & France & Netherlands \\
\hline Hulya Celebi & Francesco Giuseppe De Rosa & Mohammad Elorbany \\
\hline Turkey & Italy & USA \\
\hline Maurizio Cereda & Assunta De Vitis & Ali Elsayes \\
\hline USA & Italy & USA \\
\hline Jonathan Charnin & Giorgio Della Rocca & Mohamed El-Tahan \\
\hline USA & Italy & Egypt \\
\hline Cosimo Chelazzi & Joseph Deng & Emre Erbabacan \\
\hline Italy & USA & Turkey \\
\hline Catherine Chen & Mark Dershwitz & Ali Fuat Erdem \\
\hline USA & USA & Turkey \\
\hline Vidya Chidambaran & Andre Dewolf & Nauder Faraday \\
\hline USA & USA & USA \\
\hline
\end{tabular}


Ehab Farag

USA

David Faraoni

Belgium

Hassan Farhan

USA

Argyro Fassoulaki

Greece

John Feiner

USA

Paolo Feltracco

Italy

Karim Fikry

USA

Ryan Fink

USA

Alfonso Fiorelli

Italy

Michael Firstenberg

USA

Stuart Forman

USA

Gyorgy Frendl

USA

Inez Frerichs

Germany

Eva Fuentes

USA

Tatsuma Fukuda

Japan

Tibor Fulop

USA

Richard Galgon

USA

Samuel Galvagno

USA

Marcelo Gama De Abreu

Germany

Roser Garcia-Guasch

Spain

Marc Garnier

France

Lene Heise Garvey

Denmark
Mona Ring Gätke

Denmark

Markus Gehling

Germany

Marco Gemma

Italy

Marc Gentili

France

Mohamed Amin Ghobadifar

Iran

Chiara Giorni

Italy

Massimiliano Greco

Italy

Vadim Gudzenko

USA

Jean Guglielminotti

France

Freedom Nkhululeko Gumedze

South Africa

Dhanesh Gupta

USA

Harshad Gurnaney

USA

Thomas Hachenberg

Germany

Robert G Hahn

Sweden

Seetharaman Hariharan

Trinidad and Tobago

Erik K. Hartmann

Germany

Soshi Hashimoto

Japan

Randolph Hastings

USA

Goran Hedenstierna

Sweden

Jan Hendrickx

Belgium

Matthias Heringlake

Germany

Ross Hofmeyr

South Africa
Masaru Honda

Japan

Jean-Louis Horn

USA

Hisham Hosny

Egypt

Tim Howes

United Kingdom

Martin Huelsmann

Austria

Jennifer Hunter

United Kingdom

Jung-Won Hwang

South Korea

Luiz Eduardo Imbelloni

Brazil

Craig Jabaley

USA

Narasimhan Jagannathan

USA

Michael James

South Africa

Wei Jiang

China

Daniel Johnson

USA

Girish Joshi

USA

Rebecca Kalman

USA

Dharshi Karalapillai

Australia

Menelaos Karanikolas

USA

Brad Karon

USA

Zbigniew Karwacki

Poland

Marc Kastrup

Germany

Joel Katz

Canada

Nermin Kelebek Girgin

Turkey 
Max Kelz

USA

Hanan Khafagy

Egypt

Bodin Khwannimit

Thailand

Rainer Kiefmann

Germany

Hae-Keum Kil

South Korea

Chol Kim

Japan

Ji Young Kim

South Korea

Jong Yeop Kim

South Korea

Kristopher Kimmell

USA

Detlef Kindgen-Milles

Germany

Michel Kindo

France

Erik Kistler

USA

Kemalettin Koltka

Turkey

Ozlem Korkmaz Dilmen

Turkey

Irene Kourbeti

Greece

Morten Tange Kristensen

Denmark

Michael Alexander Kuiper

Netherlands

Eiichi Kumamoto

Japan

Alexander Kuo

USA

John Laffey

Canada

Giovanni Landoni

Italy

Meghan Lane-Fall

USA
Malinee Laopaiboon

Thailand

Asad Latif

USA

Bruno Laviolle

France

J. Adam Law

Canada

Chiara Lazzeri

Italy

Jae W Lee

USA

Po-Shun Lee

USA

Sangseok Lee

South Korea

Christian Lehmann

Canada

Hendrikus Lemmens

USA

Edmund Leung

United Kingdom

Vincent Lew

USA

Kent Lewandrowski

USA

Hsin Jung Lin

USA

Jun Lin

USA

Benigno Linares Segovia

Mexico

Michael Lipnick

USA

Steven Lisco

USA

Celia Lloret-Linares

France

Anne Lui

Canada

Nadia Lunardi

USA

Carl Lynch

USA
Christopher Lysakowski

Switzerland

Jean-Marc Malinovsky

France

Laxmaiah Manchikanti

USA

Jeff Mandel

USA

Dauri Mario

Italy

Claes-Roland Martling

Sweden

Christie Mato

Nigeria

Eckhard Mauermann

Switzerland

Tommaso Mauri

Italy

Courtney Maxey-Jones

USA

Lynne Maxwell

USA

Michael Mazzeffi

USA

Colin Mccartney

Canada

Duncan Mclean

USA

Graeme Mcleod

United Kingdom

Kamal Medlej

Lebanon

Francesco Menichetti Italy

Guniz Meyanci Koksal Turkey

Pavel Michalek

United Kingdom

Cyrus Mintz

USA

Calin Mitre

Romania

Nicholas Mohr

USA 
Hasse Møller-Sørensen

Denmark

Zsolt Molnar

Hungary

Andrea Montisci

Italy

Ingrid Moreno Duarte

USA

Rory Morty

Germany

Jeremi Mountjoy

USA

George Mychaskiw

USA

Yasuko Nagasaka

USA

Bala Nair

USA

Giuseppe Natalini

Italy

Vladimir Nekhendzy

USA

Kelly Newman

USA

Pornswan Ngamprasertwong

USA

Warwick Ngan Kee

China

\section{Albert Nguyen}

USA

David Nicolau

USA

Kamilla Nielsen

Denmark

Diederik Nieuwenhuijs

Netherlands

Catherine Nix

Ireland

Marko Noc

Slovenia

Anders Kehlet Nørskov

Denmark

Mohamed Nour

USA
Ala Nozari

USA

Omar Omar

USA

Andrea Orfanakis

USA

Sisse R Ostrowski

Denmark

Megan Othus

USA

Yen-Chuan Ou

Taiwan

Mehmet Ozcan

USA

Dilek Ozcengiz

Turkey

Peter Paal

Austria

Sher-Lu Pai

USA

Krishna Parekh

USA

Martyn Parker

United Kingdom

Pisano Pascale

France

Maurizio Passariello

Italy

Amit Patel

USA

Kashyap Patel

Australia

Piyush Patel

USA

Vittorio Pavoni

Italy

Jason Peart

Australia

Paolo Pelosi

Italy

Yingwei Peng

Canada

Pernille Lykke Petersen

Denmark
Tobias Piegeler

Switzerland

Marina Pieri

Italy

Annop Piriyapatsom

USA

Massimiliano Pirrone

Italy

Jean-Francois Pittet

USA

Valentina Porta

Brazil

Alen Protic

Croatia

Prakash P Punjabi

United Kingdom

Lorenzo Quario Rondo

Italy

Douglas Raines

USA

Saifudin Rashiq

Canada

Lars Rasmussen

Denmark

Kristofer Rau

USA

James Rhee

USA

Paul Riegelhaupt

USA

Hynek Riha

Czech Republic

Joseph Rinehart

USA

Katherine Robinson

Australia

Antonio Rodríguez Núñez

Spain

Dae-Hyun Roh

South Korea

Stefano Romagnoli

Italy

Carl Rosow

USA 
Kenneth Rothfield
USA

Gabriel Rusanescu

USA

\begin{abstract}
Alisdair Ryding
United Kingdom

Jung-Hee Ryu

South Korea
\end{abstract}

Mohammad Ali Sahmeddini

Iran

Sam Salman

Australia

Adam Sapirstein

USA

Jana Sawynok

Canada

Thomas Scheeren

Netherlands

Christoph Schlimp

Austria

Andre Schmidt

Brazil

Ulrich Schmidt

USA

Andreas Schneider

Germany

Tobias Schuerholz

Germany

Stephan Schug

Australia

Peter Schulman

USA

Marcus J Schultz

Netherlands

Kurt Schumacher

USA

Wolfram Schummer

Germany

Martin Schuster

Germany

Lalit Sehgal

India

Nuzhet Mert Senturk

Turkey
Ary Serpa Neto

Netherlands

Michael Serpell

United Kingdom

You Shang

China

Reza Shariat Moharari

Iran

Kenneth Shelton

USA

Shiqian Shen

USA

Koh Shingu

Japan

Matthew Sigakis

USA

Paolo Silvani

Italy

Brett Simon

USA

David Simpson

USA

Aparna Sinha

India

Ashish Sinha

USA

Sarath Sistla

India

Markus Skrifvars

Finland

Andreas Skyschally

Germany

Callum Sleigh

New Zealand

Anja Slikkerveer

Netherlands

Mieke Soens

USA

Sohan Solanki

India

Xue-Jun Song

USA

Massimiliano Sorbello

Italy
Philip Spencer

USA

Peter Spieth

Germany

Vanessa Stadlbauer

Austria

David Stahl

USA

Joel Starkopf

Estonia

Stanislaw Stawicki

USA

Bernd Stegmayr

Sweden

Kenan Stern

USA

Francesca Stoppa

Italy

Bala Subramaniam

USA

Rajeev Subramanyam

USA

Takahiro Suzuki

Japan

Andrea Székely

Hungary

Szilard Szucs

Ireland

Paul Szumita

USA

Christopher Tainter

USA

Boyd Taylor Thompson

USA

Anders Thorell

Sweden

Kevin Thornton

USA

Yuke Tian

China

Kevin Tremper

USA

Tanja Treschan

Germany 
Luigi Tritapepe

Italy

Melek Tulunay

Turkey

Andrew Udy

Australia

Basak Ugurlu

Turkey

Susana Vacas

USA

Tom Van Der Poll

Netherlands

Pieter Van Der Starre

USA

Arthur Van Zanten

Netherlands

Atul Vats

USA

Nicholas Ventham

United Kingdom

Marcel Vercauteren

Belgium

Tomas Vymazal

Czech Republic

Alexander Wahba

Norway

Samuel Wald

USA

Fuzhou Wang

China

Marcin Wasowicz

Canada
Timothy Webb

USA

Joseph Weidman

USA

Avi Weinbroum

Israel

Toby Weingarten

USA

Nicolas Weiss

France

Tyler Wellman

USA

Elizabeth Whitlock

USA

Christoph Wiese

Germany

Craig Williamson

USA

David Williamson

Canada

Cynthia Wong

USA

Hector Wong

USA

Hermann Wrigge

Germany

Hinnerk Wulf

Germany

Christian Wunder

Germany

Hannah Wunsch USA
Emre Yalcinkaya

Turkey

Bin Yang

United Kingdom

Daniel Dante Yeh

USA

Jun Rho Yoon

South Korea

Nayer Youssef

Canada

Ahmed Zaafran

USA

Massimo Zambon

Italy

Alberto Zanella

Italy

Christine Zanghi

USA

Emanuela Zannin

Italy

Valerie Zaphiratos

Canada

Nick Zavras

Greece

Fatih Zor

Turkey

Martin Zoremba

Germany

Received: 13 January 2015 Accepted: 13 January 2015 Published: 6 February 2015

doi:10.1186/1471-2253-15-10

Cite this article as: Rowles: BMC

Anesthesiology reviewer acknowledgement,

2014. BMC Anesthesiology 2015 15:10. 